\title{
How to estimate the distance to the warm absorber in AGN from photoionized models
}

\author{
A. Różańska ${ }^{1}$, I. Kowalska ${ }^{2}$, and A. C. Gonçalves ${ }^{3,4,5}$ \\ 1 Copernicus Astronomical Center, Bartycka 18, 00-716 Warsaw, Poland \\ e-mail: agata@camk.edu.pl \\ 2 Warsaw University Observatory, Al. Ujazdowskie 4, 00-478 Warsaw, Poland \\ 3 Observatoire Astronomique de Strabsourg, Université Louis Pasteur, CNRS, 11 rue de l'Université, 67000 Strasbourg, France \\ 4 LUTH, Observatoire de Paris, CNRS, Université Paris Diderot, 5 place Jules Janssen, 92190 Meudon, France \\ 5 CAAUL, Observatório Astronómico de Lisboa, Tapada da Ajuda, 1349-018 Lisboa, Portugal
}

Received 8 February 2008 / Accepted 22 May 2008

\section{ABSTRACT}

\begin{abstract}
Aims. We present a method that allows us to estimate the distance from the continuum source located in the center of AGN to the highly ionized gas called warm absorber.

Methods. We computed a set of constant total pressure photoionization models compatible with the warm absorber conditions, where a metal-rich gas is irradiated by a continuum in the form of a double power law. The first power law is hard up to $100 \mathrm{keV}$ and represents radiation from an X-ray source, while the second power law extends up to several eVs and illustrates radiation from an accretion disk.

Results. When the ionized continuum is dominated by the soft component, the warm absorber is heated by free-free absorption, instead of Comptonization, and the transmitted spectra show different absorption line characteristics for different values of the hydrogen number density at the cloud illuminated surface.

Conclusions. This fact results in the possibility of deriving the number density on the cloud illuminated side from observations and hence the distance to the warm absorber.
\end{abstract}

Key words. Atomic processes - radiative transfer - galaxies: active - ultraviolet: galaxies - X-rays: galaxies

\section{Introduction}

Many active galactic nuclei (AGN) exhibit numerous absorption features of highly ionized material in their UV/X-ray spectra. Such gas, called warm absorber (hereafter WA), is located on the line-of-sight towards the observer and it is illuminated by radiation originating from the active nucleus.

Most of the observed absorption lines are blushifted, suggesting that the metal-rich gas is outflowing. We still do not know how such a wind is powered and where it is launched, nevertheless, due to high-resolution spectroscopic observations of AGN from FUSE, Chandra, XMM, and other satellites, we were able to make some diagnostics on the physical conditions of the WA (for a review see: Kriss 2004; Blustin et al. 2005; Różańska 2007).

The observed absorption lines velocity shifts are close to $10^{4} \mathrm{~km} \mathrm{~s}^{-1}$ in the case of UV absorbers (Gabel et al. 2003) and around $10^{2}$ to $10^{3} \mathrm{~km} \mathrm{~s}^{-1}$ in the case of X-rays (Kaspi et al. 2001; Kaastra et al. 2002). The column density of the WA is generally estimated to be about $10^{21-23} \mathrm{~cm}^{-2}$. The absorbing gas comprises different ionization phases, corresponding to temperatures from about $10^{5} \mathrm{~K}$, when iron is partially ionized, up to a few $10^{7} \mathrm{~K}$, when iron is almost completely ionized (Netzer et al. 2003; Krongold et al. 2003; Steenbrugge et al. 2005). From the above hints, photoionization modeling of the WA is done, trying to answer to the most important and unsolved question: how far from the continuum source is the absorbing gas located?

However, there is one difficulty that prevents us from answering this question. It is well known that photoionized models of a cloud illuminated by a single X-ray power law, typically ranging from $0.01 \mathrm{keV}$ up to $100 \mathrm{keV}$ (hereafter we call this a hard X-ray illumination) are degenerate (Różańska 2007). We cannot distinguish between clouds with the same ionization parameter, but different hydrogen number density $n_{0}$, at the cloud illuminated surface and at different locations. The transmission spectrum from a rarefied cloud $\left(n_{0} \sim 10^{5} \mathrm{~cm}^{-3}\right)$ located farther away i.e. at $\sim 0.1 \mathrm{pc}$, is identical to the spectrum of a dense cloud $\left(n_{0} \sim 10^{10} \mathrm{~cm}^{-3}\right)$ situated at $\sim 0.0001 \mathrm{pc}$ from a continuum source. The first case is consistent with the WA being co-spatial with the NLR (narrow line region) or dusty torus, while the second case corresponds to the closest neighborhood of an accretion disk and BLR (broad line region).

Some estimations of the distance to the WA were done using variability studies (Netzer et al. 2003; Krongold et al. 2005). However, using the same $900 \mathrm{ksec}$ Chandra data of NGC 3783, Netzer et al. (2003) found upper limits for the location of three ionization phases to be $3.2,0.63$, and $0,18 \mathrm{pc}$, respectively, while Krongold et al. (2005) estimated the location of the high ionization phase at $0.0029 \mathrm{pc}$ and low ionization phase at 0.0004-0008 pc.

In this paper we show that the degeneracy of photoionized models breaks down when the WA is illuminated by an AGN continuum including the thermal disk radiation, represented here as a second power-law component. The disk contribution is particularly important in the case of quasars, since their broad band spectra are clearly dominated by the soft disk emission. In this article we explain why double power-law models are not 
degenerate and claim that using such models provides a way to determine the distance to the WA in some objects.

The structure of the paper is as follows. In Sect. 2 we explain how to estimate the distance to the WA from photoionized models. Section 3 describes the photoionization models used in this work. Results for different spectral shapes are presented in Sect. 4. Discussion of the models is presented in Sect. 5, and main conclusions of our work are drawn in Sect. 6.

\section{The distance to the warm absorber}

The distance from the central engine to the WA is a clue issue for understanding the wind geometry. We can derive this distance from observations using photoionized models, which are parametrized by the so-called ionization parameter, defined at the cloud surface. There are three ionization parameters being used by different groups and photoionization codes, but the idea is the same: to define the amount of ionized radiation that reaches the cloud. Here, we discuss the problem of distance derivation using the ionization parameter defined as

$\xi=\frac{L_{\text {ion }}}{n R^{2}}$,

where $L_{\text {ion }}$ is the ionizing source luminosity, $n$ the hydrogen number density, and $R$ the distance from an illuminating source. Nevertheless, the problem of distance derivation does not depend on the ionization parameter used by different groups, and it refers to the value of this parameter on the cloud surface i.e. for intrinsic luminosity and for surface hydrogen number density, hereafter $n_{0}$.

From observations, we are able to get the luminosity of the object as a separate measurement, and the ionization parameter on the cloud surface is obtained by fitting the data with a given photoionization model. But we are still left with the $n_{0} R^{2}$ product, meaning the distance to the WA depends on the value of the number density at the cloud illuminated surface.

Below, we show how to determine the number density at the illuminated side of the WA using photoionization modeling. Then, after simple inversion of Eq. (2), we obtain the distance to the absorber.

\section{Photoionization modeling}

In this paper all photoionization models are computed using the TITAN code developed by Dumont et al. (2000); Collin et al. (2004). TITAN is well-suited to the study of both optically thick and thin media, such as the WA. It computes the gas structure in thermal and ionization equilibrium assuming non-LTE, and it provides the reflected, emitted outward, and absorbed spectra. TITAN treats the transfer of both the lines and the continuum using the ALI (accelerated lambda iteration) method, which precisely computes line and continuum fluxes in a self-consistent way. Our atomic data include $\sim 10^{3}$ lines from ions and atoms of $\mathrm{H}, \mathrm{He}, \mathrm{C}, \mathrm{N}, \mathrm{O}, \mathrm{Ne}, \mathrm{Mg}, \mathrm{Si}, \mathrm{S}$, and $\mathrm{Fe}$, all elements having cosmic abundances.

The total (gas + radiation) pressure is computed after the plasma temperature has been determined by the thermal balance equation. The pressure is kept constant within the medium, thus allowing the density profile to be determine. The model is iterated until convergence. We have shown that the assumption of constant pressure allows the ionized gas to be naturally stratified due to illumination (Różańska et al. 2006; Gonçalves et al. 2006); as a result, the cloud comprises different ionization stages, which is consistent with observations.
In all our models we assume a plane-parallel geometry, with the WA medium illuminated on one side by a given incident continuum. We assume the external radiation to hit the cloud perpendicular to its surface.

In the frame of photoionization modeling of the warm absorber, there is no possibility of reconstructing velocity structure observed in some cases (e.g. Steenbrugge et al. 2005), where each ion has a slightly different velocity. We can only consider the case where all ions have the same turbulent velocity, and it is taken into account in our code.

The models are parametrized by the ionization parameter $\xi$ at the cloud surface, the total column density $N_{\mathrm{H}}$, the hydrogen number density at the cloud illuminated surface $n_{0}$, and the incident flux spectral energy distribution (SED).

The parameter $\xi$ is very well-constrained on the surface of an illuminated medium. But if we want to trace ionization properties deeper in the gas, we use the so-called dynamical ionization parameter defined as

$\Xi=\frac{\xi}{4 \pi c k T}=\frac{L_{\mathrm{ion}}}{4 \pi c R^{2}} \frac{1}{n k T}=\frac{F_{\mathrm{ion}}}{c P_{\mathrm{gas}}}=\frac{P_{\mathrm{rad}}}{P_{\mathrm{gas}}}$,

where $c$ is a velocity of light, $T$ temperature of the gas, $F_{\text {ion }}$ a flux which affects the cloud, and $P_{\text {rad }}$ and $P_{\text {gas }}$ are radiation and gas pressure, respectively. Below, we describe the ionization structure of presented clouds using relation $\Xi(T)$.

In this paper we consider two possible shapes of SED first: a single power law roughly representing the spectra of Seyfert galaxies, and second, a double power law representing the spectra of disk dominated bright quasars. In both cases, the power laws are modified by exponential high- and low-energy cut-offs according to the formula:

$F_{E}=E^{-\alpha} \exp \left(-E / E_{\max }\right) \exp \left(-E_{\min } / E\right)$,

where $F$ is the flux, $E$-energy, $\alpha$-spectral index, and $E_{\min }$ and $E_{\max }$ are low and high spectral ranges, respectively. In the case of double power-law models, we have an additional parameter, which is the relative normalization of both power-law components, i.e. the ratio of their integrated fluxes denoted as $F_{X} / F_{\text {soft }}$. This relative normalization and the exponential cut-offs ensure that there is no discontinuity at the point where two power-laws merge.

\section{Results for the different shape of the illuminated continuum}

To illustrate the determination of distance by photoionized models, we consider three clouds with different hydrogen density numbers at the illuminated surface: $n_{0}=10^{6}, 10^{8}$, and $10^{10} \mathrm{~cm}^{-3}$. The ionization parameter on the cloud surface is the same in the three cases and $\xi=10^{5}$. The total column density of each cloud is taken to be close to the maximum column density for which the temperature profile is thermally stable for a given spectral energy distribution (see Różańska 2007). In all cases, the total column density is on the order of $10^{23} \mathrm{~cm}^{-2}$, and we assume the turbulent velocity of the gas equaled $300 \mathrm{~km} \mathrm{~s}^{-1}$.

For a single power-law irradiation, the primary continuum spectrum ranges from 0.01 up to $100 \mathrm{keV}$ and has a spectral index equal to 0.8 . In the case of a double power-law, the softer power law ranges from $10^{-7}$ up to $0.0136 \mathrm{keV}$ with a spectral in$\operatorname{dex} \alpha_{\text {soft }}=0.3$, and the hard continuum component has a spectral index $\alpha_{X}=0.8$, ranging from 0.0136 up to $100 \mathrm{keV}$. We consider three cases of relative normalization of both power-law components: (i) $F_{X} / F_{\text {soft }}=0.01$, corresponding to a domination 

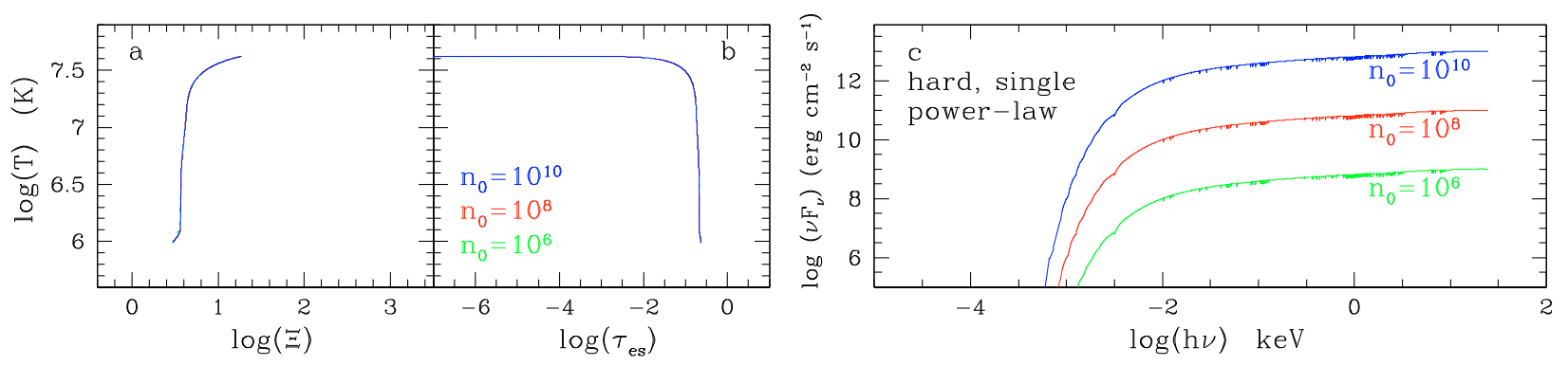

Fig. 1. Ionization structure (a), temperature structure (b), and transmitted spectrum (c) for a WA illuminated by a hard single power-law continuum for three different values of $n_{0}: 10^{6} \mathrm{~cm}^{-3}$ (green line), $10^{8} \mathrm{~cm}^{-3}$ (red line), and $10^{10} \mathrm{~cm}^{-3}$ (blue line).
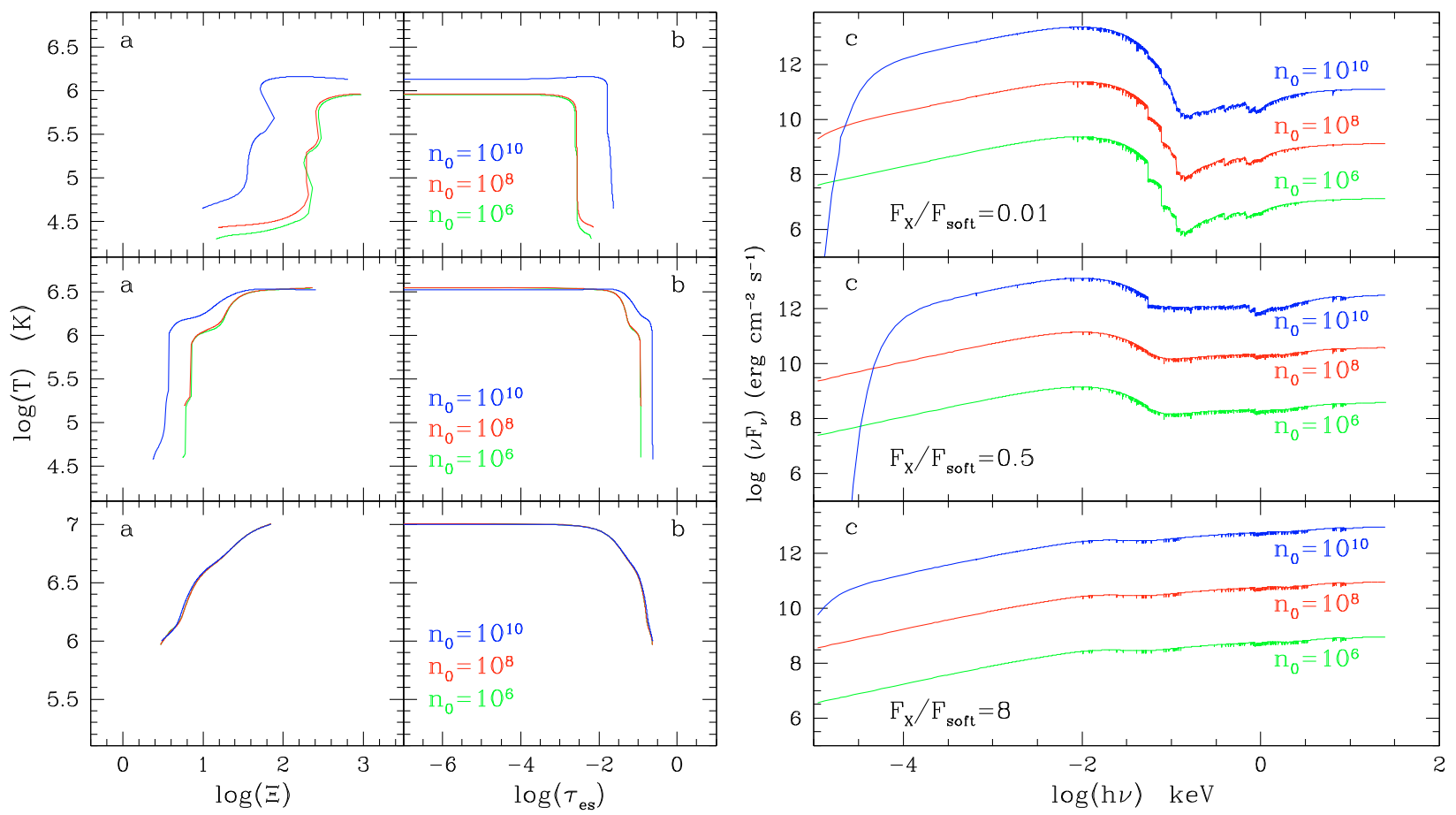

Fig. 2. Ionization structures (a), temperature structures (b), and transmitted spectra (c) for the WA illuminated by a double power-law for three different values of $n_{0}: 10^{6} \mathrm{~cm}^{-3}$ (green line), $10^{8} \mathrm{~cm}^{-3}$ (red line), and $10^{10} \mathrm{~cm}^{-3}$ (blue line). Upper panels correspond to $F_{X} / F_{\text {soft }}$ equal 0.01, middle panels to 0.5 , and bottom panels to 8 .

by the soft component; (ii) $F_{X} / F_{\text {soft }}=0.5$, typical of an intermediate case; and (iii) $F_{X} / F_{\text {soft }}=8$, where the hard component dominates.

\subsection{Hard, single power-law photoionized models}

The comparison of the clouds with different $n_{0}$ values irradiated by a hard, single power law is presented in Fig. 1. Panel a represents the ionization structure through the WA, which is identical for all $n_{0}$ values. The maximum temperature on the illuminated side of the clouds is quite high, on the order of $4 \times 10^{7} \mathrm{~K}$. Going deeper into the WA, the temperature structure displays a strong gradient (panel b), which is consistent with the gas structure being in hydrostatic equilibrium and illuminated by hard X-rays (Różańska et al. 2002; Madej \& Różańska 2004).

The transmitted spectra are presented in Fig. 1c. They differ only in normalization, which means that the low density cloud, with $n_{0}=10^{6} \mathrm{~cm}^{-3}$, located farther away from the continuum source (see Eq. (2)), has the same spectral features as the dense cloud of $n_{0}=10^{10} \mathrm{~cm}^{-3}$, located two orders of magnitude closer to the source. The detailed comparison of lines for those two cases is presented in Fig. 5 (bottom panels). Lines are identical in all spectral bands; therefore, models with a single, hard power-law illumination are highly degenerate and useless for the purpose of determining the distance to the WA.

\subsection{Double power-law photoionized models}

The situation changes when we consider a primary continuum described by a double power law dominated by its soft component. In Fig. 2 we show results for clouds illuminated by a double power law with increasing $F_{X} / F_{\text {soft }}$ ratio, from 0.01 (upper panel) up to 8 (bottom panel). When the continuum soft component dominates, the ionization and temperature structures of the considered clouds differ for different values of $n_{0}$. Separation between models is clearer for lower values of the $F_{X} / F_{\text {soft }}$ ratio. 


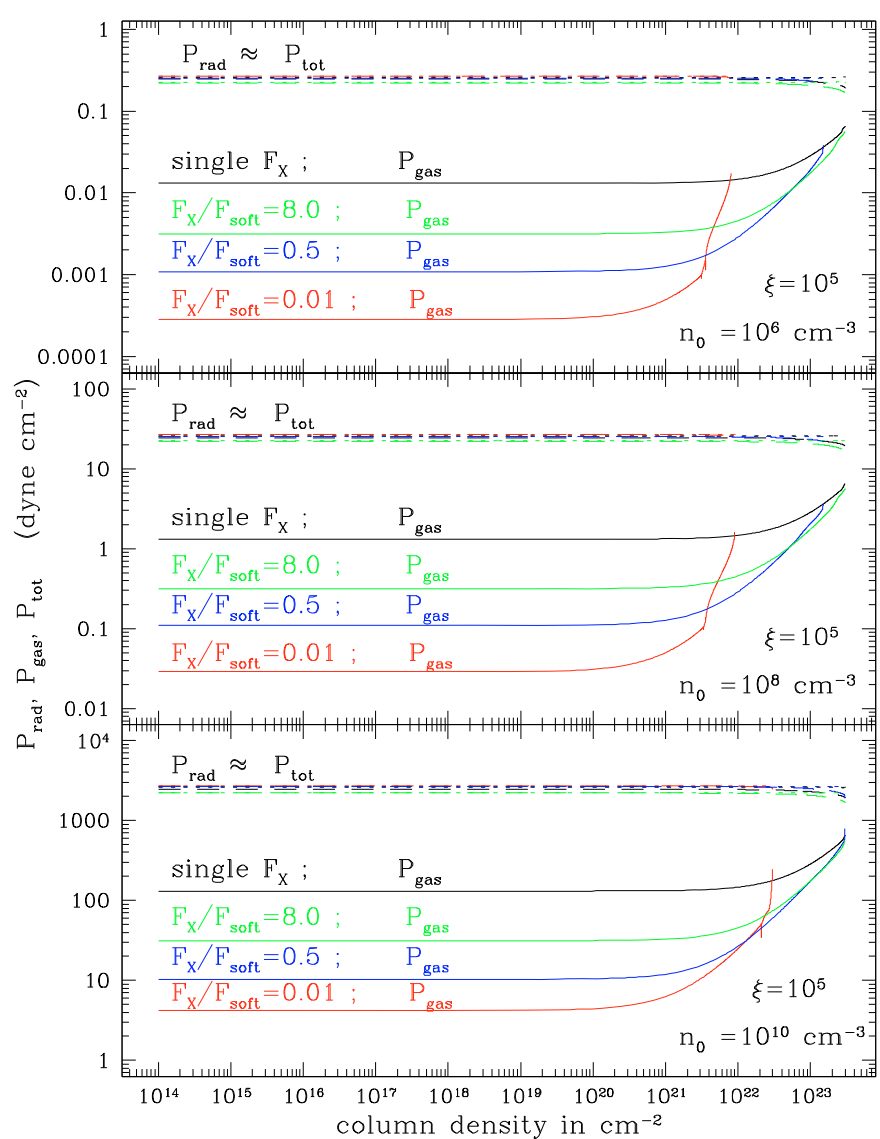

Fig. 3. Comparison of the gas pressure (solid line), the radiation pressure (dashed line), and the total, i.e. gas + radiation, pressure (dotted line), for all computed models. The upper panel corresponds to clouds with $n_{0}=10^{6} \mathrm{~cm}^{-3}$, the middle panel to $n_{0}=10^{8} \mathrm{~cm}^{-3}$, and the bottom panel to $n_{0}=10^{10} \mathrm{~cm}^{-3}$.

The equilibrium temperature at the illuminated face of the cloud is about one order of magnitude lower than in the case of a single power-law illumination.

For the highest value of $n_{0}=10^{10} \mathrm{~cm}^{-3}$, the continuum spectrum displays a significant absorption in the low energy band due to free-free absorption. Looking closer at the transmitted spectra, there is a big difference in the equivalent width (EW) of absorption lines between clouds of two extreme values of $n_{0}$, and for $F_{X} / F_{\text {soft }}=0.01$; this is illustrated in Fig. 5 upper panels. For instance, $\mathrm{EWs}$ of $\mathrm{HO}_{\delta}$ and $\mathrm{HO}_{\epsilon}$ lines for the case of $n_{0}=10^{10} \mathrm{~cm}^{-3}$ are ten times smaller than for the case of $n_{0}=10^{6} \mathrm{~cm}^{-3}$. In X-ray domain, EWs of the sample lines: $\mathrm{Fe} 19_{\delta}, \mathrm{Fe} 20_{\delta}, \mathrm{Fe} 17_{f l}$ and $\mathrm{Fe} 19_{f l}$ are respectively seven, five, fourteen, and four times more for the cloud with $n_{0}=10^{10} \mathrm{~cm}^{-3}$ than for the cloud with $n_{0}=10^{6} \mathrm{~cm}^{-3}$. Therefore, from the ratios of EWs of absorption lines alone, we can select one particular photoionized model that fits the observations; then having obtained the number density at the illuminated cloud surface, we can derive the distance to the WA.

The observed differences of photoionized models can be much greater for different spectral energy distributions as shown by Różańska (2007). Below, we describe what causes these differences in the case of a WA.

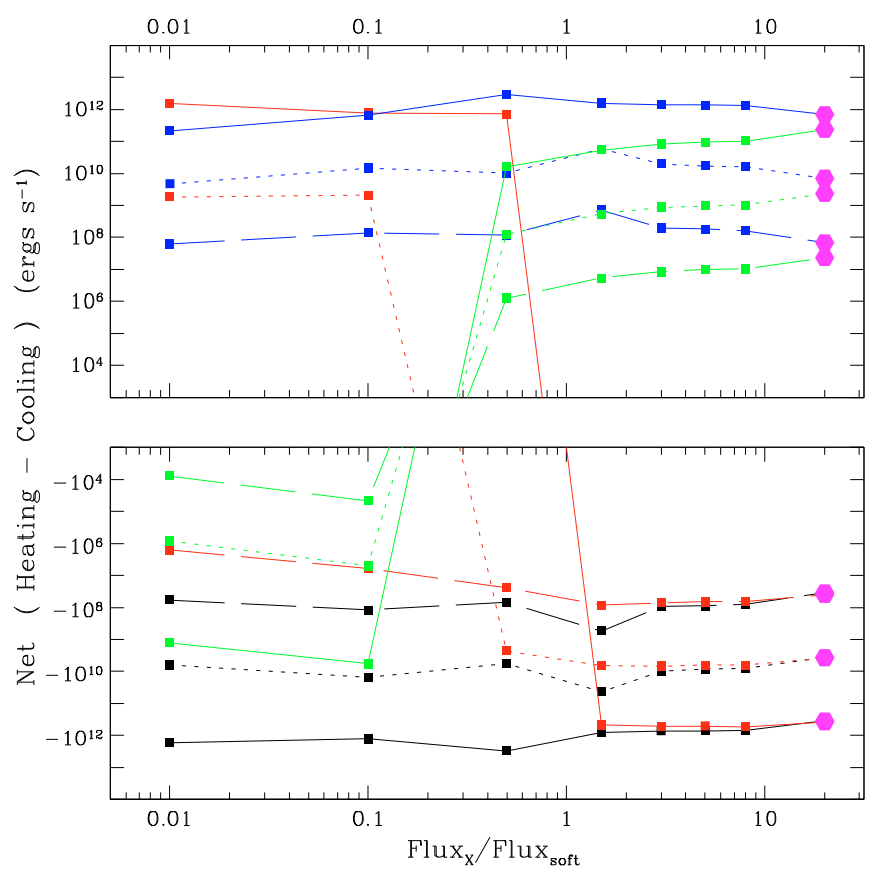

Fig. 4. Net, i.e. Heating-cooling gains of energy integrated over the cloud versus the ratio of hard X-rays to the soft power-law component. Net gains for Comptonization are represented by green lines, boundfree processes, i.e. ionization-recombination, are represented by blue lines, bound-bound processes, i.e. line heating-cooling, by black lines, and free-free processes by red lines. Since Axies are logarithmic, we therefore arbitrarily assigned a minus sign to those cases where total gains are negative, i.e. cooling dominates over heating (bottom panel). There is a discontinuity between the cases where heating dominates over cooling (upper panel). For each process, solid lines represents clouds with $n_{0}=10^{10} \mathrm{~cm}^{-3}$, dotted lines $n_{0}=10^{8} \mathrm{~cm}^{-3}$, and dashed lines $n_{0}=10^{6} \mathrm{~cm}^{-3}$. Magenta hexagons mark gains for the case of a hard, single power-law illumination.

\section{Discussion}

To understand why single power-law models are degenerate and why models can be effectively separated after including a second illuminating component, we analyzed of the basic physical conditions inside the clouds. We followed the behavior of the parameters responsible for the hydrostatic and the ionized thermal equilibrium of the gas, i.e. pressure and efficiency of radiative processes.

\subsection{Pressure balance through the cloud}

All computed cloud models are dominated by radiation pressure, as shown in Fig. 3. For all cases, the radiation pressure practically equals the total (gas + radiation) pressure. There is nothing unusual in the behaviour of the gas pressure for all computed models. For a given $n_{0}$ the gas pressure is highest for the highest $F_{X} / F_{\text {soft }}$. Obviously, the value of the gas pressure on the cloud surface is proportional to the value of the hydrogen number density on the cloud illuminated side.

\subsection{Energy balance within the cloud}

The TITAN code solves the energy balance by calculating radiation gains and losses at each point of the gas slab. Figure 4 shows the net heating minus cooling rate integrated over the whole cloud versus the ratio of the two continuum components, 

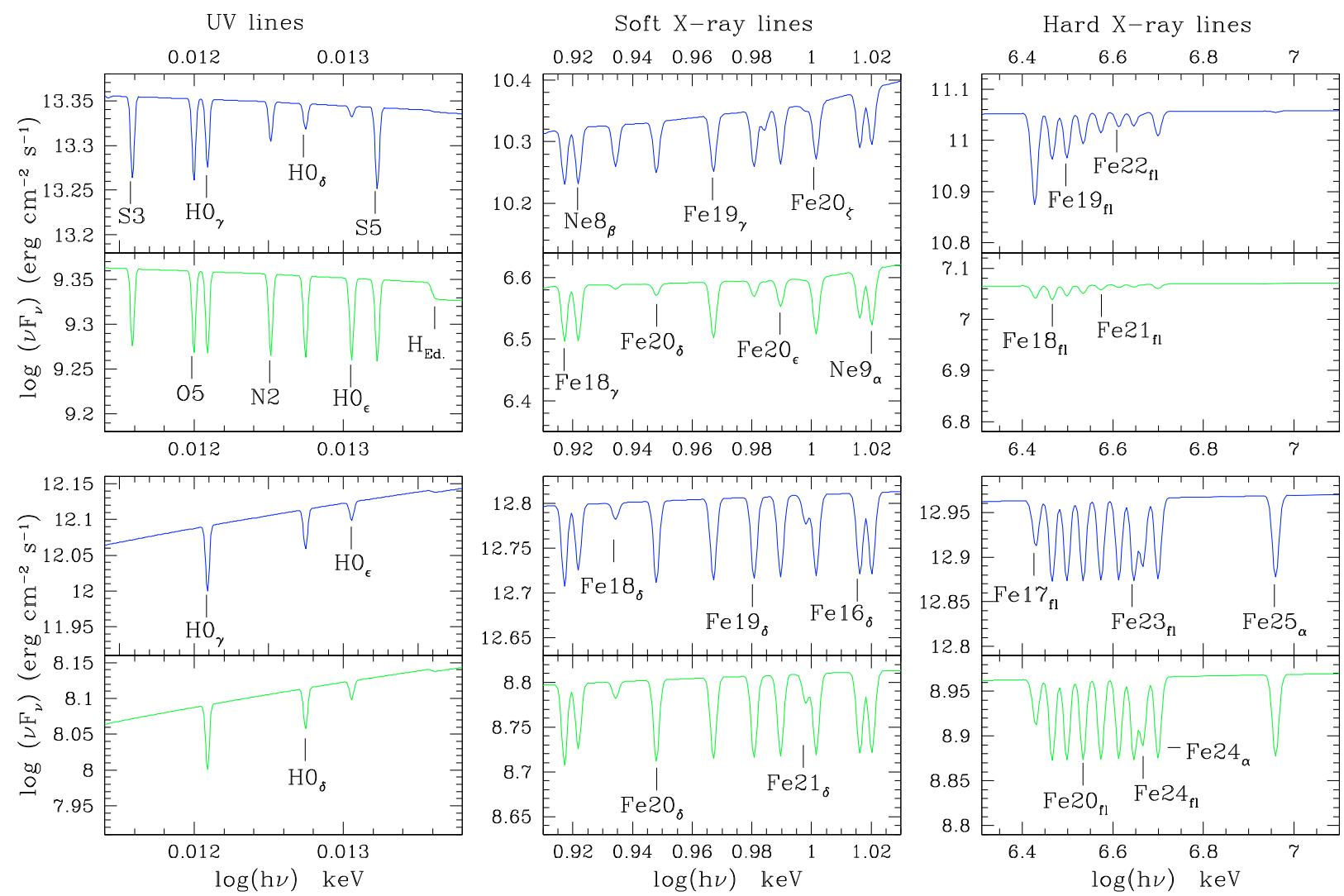

Fig. 5. Comparison of spectral absorption lines for three energy bands: UV (left panels), soft X-ray (middle panels), and hard X-ray lines (right panels). Upper panels show the case where the WA is illuminated by a double power law with $F_{X} / F_{\text {soft }}=0.01$, while the bottom panels correspond to irradiation by a hard, single power law. Blue lines depict dense clouds of $n_{0}=10^{10} \mathrm{~cm}^{-3}$, and green lines less dense clouds of $n_{0}=10^{6} \mathrm{~cm}^{-3}$.

$F_{X} / F_{\text {soft }}$. Each important mechanism was analyzed separately to identify its contribution to the total energy balance in the WA.

The heating and cooling rates cover a significant range, so we decided to plot the results on a logarithmic scale. We arbitrarily assigned a minus sign to those cases where total gains are negative, i.e. when cooling dominates (bottom panel). The sign is positive when heating dominates (upper panel). There is an arbitrarily discontinuity between the two panels in Fig. 4, which does not have any physical meaning. A different type of line is used to denote clouds of different number densities, $n_{0}=10^{10} \mathrm{~cm}^{-3}$ (solid line), $n_{0}=10^{8} \mathrm{~cm}^{-3}$ (dotted line), and $n_{0}=10^{6} \mathrm{~cm}^{-3}$ (dashed line). Magenta hexagons represent the net heating minus cooling for the case of a single power-law illumination, they are obvious limits of the radiation rates when increasing the $F_{X} / F_{\text {soft }}$ ratio.

Hard X-ray radiation always heats the WA, even if the X-ray component is very weak. The same is true for the line cooling, which most probably is balanced by photoionization. The situation changes dramatically for Comptonization and free-free processes. For $F_{X} / F_{\text {soft }}$ decreasing from 1 and 0.1, Compton heating decreases and the WA gas starts to even be Compton cooled by soft disk photons. The same happens in the case of bremsstrahlung free-free processes. Bremsstrahlung heating starts to dominate when Compton heating becomes unimportant. This means that, in the case of a WA illuminated by a double power law, the presence of a soft, disk component changes the mechanism responsible for the high temperature equilibrium on the irradiated side of the cloud. Therefore, in such a situation, clouds are heated mostly by bremsstrahlung and not by
Comptonization, as it is in cases of clouds illuminated by a single, hard X-ray continuum.

\subsection{Absorption lines from double power-law models}

In Fig. 5, we focus on the absorption lines present in three spectral bands for clouds with $n_{0}=10^{10} \mathrm{~cm}^{-3}$ (blue line) and $n_{0}=10^{6} \mathrm{~cm}^{-3}$ (green line). The upper panels represent models with $F_{X} / F_{\text {soft }}=0.01$, while the bottom panels depict models of clouds illuminated by a single, hard X-ray power law.

For strongly degenerate models, the absorption line spectra are identical and there is no possibility of distinguishing them based on the number density $n_{0}$ just by fitting photoionization models to the observations. The situation is very different in the case of a double power-law illumination. The equivalent widths of some absorption lines differ for different $n_{0}$, giving the possibility of fitting one single photoionization model to the observed spectrum. Therefore, having a grid of photoionized models providing the main absorption line ratios, we can estimate the number density at the cloud illuminated surface, and thus the distance to the WA. Indeed, even modeling only the X-ray data of any AGN, we have to take into account that the WA is affected not only by an X-ray power law from the corona but also by a soft component from the disk. This is accounted for in this article and justifies the use of a double power law extending from $10^{-7}$ to $100 \mathrm{keV}$. 


\section{Conclusions}

Photoionized models of a WA usually assume that the illuminating continuum has the shape of a single power law with the spectral index derived from X-ray observations of a particular AGN. In this paper, we have shown that it is important to include the broad-band continuum from the active nucleus to achieve a proper modeling of the transmission spectrum through the ionized WA gas.

We computed a set of models assuming illumination of the metal-rich material by a double power-law incident continuum, where one component mimics the emission from an accretion disk, and the second component represents the hard X-ray emission coming most probably from a hot corona or magnetic flare. This allows to break the degeneracy generally observed in photoionization models using a single hard X-ray illumination. By breaking this degeneracy we observe that models display different ionization and temperature structures for clouds with different number densities at the illuminated surface. Therefore, transmitted spectra look different and in principle it should be possible to identify the photoionized model that fits a given observation better.

We have shown that this degeneracy breaks down due to a switch in the radiative mechanism heating the ionized cloud when $F_{X} / F_{\text {soft }}$ decreases. For a single power-law irradiation, hard photons mostly participate in the Compton heating, and this process becomes responsible for the hot equilibrium temperature of the illuminated layers. When $F_{X} / F_{\text {soft }}$ decreases, i.e. when the soft component starts to dominate, Compton heating is not efficient anymore, since the cloud begins to be more efficiently cooled by Comptonization, and a hot temperature equilibrium is established due to bremsstrahlung absorption.
In conclusion, by taking broad band illumination from the active nucleus into account it is possible to determine the number density at the illuminated side of WA and thus its distance from the irradiating continuum source. This method is complementary to the method based on variability as presented by Netzer et al. (2003) and Krongold et al. (2005), and it can be applied to bright quasars with a strong disk component.

Acknowledgements. We thank Hagai Netzer, Anne-Marie Dumont, and Bożena Czerny for helpful discussions. This work was supported by the Polish Committee for Scientific Research grant No. 1 P03D 008 29, by FCT grant BPD/11641/2002, and was carried out within the framework of the European Associated Laboratory "Astrophysics Poland-France".

\section{References}

Blustin, A. J., Page, M. J., Fuerst, S. V., Branduardi-Raymont, G., \& Ashton, C. E. $2005, A \& A, 431,111$

Collin, S., Dumont, A.-M., \& Godet, O. 2004, A\&A, 419, 877

Dumont, A.-M., Abrassart, A., \& Collin, S. 2000, A\&A, 357, 823

Gabel, J. R., Crenshaw, D. M., Kraemer, S. B., et al. 2003, ApJ, 583, 178

Gonçalves, A. C., Collin, S., Dumont, A.-M., et al. 2006, A\&A, 451, L23

Kaastra, J. S., Steenbrugge, K. C., Raassen, A. J. J., et al. 2002, A\&A, 386, 427 Kaspi, S., Brandt, W. N., Netzer, H., et al. 2001, ApJ, 554, 216

Kriss, G. A. 2004, in The Interplay Among Black Holes, Stars and ISM in Galactic Nuclei, ed. T. Storchi-Bergmann, L. C. Ho, \& H. R. Schmitt, IAU Symp., 222, 223

Krongold, Y., Nicastro, F., Brickhouse, N. S., et al. 2003, ApJ, 597, 832

Krongold, Y., Nicastro, F., Brickhouse, N. S., Elvis, M., \& Mathur, S. 2005, ApJ, 622,842

Madej, J., \& Różańska, A. 2004, MNRAS, 347, 1266

Netzer, H., Kaspi, S., Behar, E., et al. 2003, ApJ, 599, 933

Różańska, A. 2007, in The Multicolored Landscape of Compact Objects and their Explosive Origins, AIP Conf. Ser., 924, 836

Różańska, A., Dumont, A.-M., Czerny, B., \& Collin, S. 2002, MNRAS, 332, 799

Różańska, A., Goosmann, R., Dumont, A.-M., \& Czerny, B. 2006, A\&A, 452, 1 Steenbrugge, K. C., Kaastra, J. S., Crenshaw, D. M., et al. 2005, A\&A, 434, 569 